\title{
Nanoscale
}

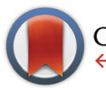

CrossMark

\&click for updates

Cite this: Nanoscale, 2015, 7, 15971

Received 10th June 2015,

Accepted 18th August 2015

DOI: 10.1039/c5nr03827h

www.rsc.org/nanoscale

\section{Anisotropic responsive microgels with tuneable shape and interactions $\dagger$}

\author{
Jérôme J. Crassous, * Adriana M. Mihut, Linda K. Månsson and Peter Schurtenberger
}

Highly monodisperse polystyrene/poly( $N$-isopropylmethacrylamide) (PS-PNIPMAM) core-shell composite microgels were synthesized and further nanoengineered in either ellipsoidal, faceted or bowl-shaped particles. Beside their anisotropy in shape, the microgel design enables an exquisite control of the particle conformation, size and interactions from swollen and hydrophilic to collapsed and hydrophobic using temperature as an external control variable. The post-processing procedures and the characterization of the different particles are first presented. Their potential as model systems for the investigation of the effects of anisotropic shape and interactions on the phase behavior is further demonstrated. Finally, the self-assembly of bowl-shaped composite microgel particles is discussed, where the temperature and an external AC electric field are employed to control the interactions from repulsive to attractive and from soft repulsive to dipolar, respectively.

\section{Introduction}

Anisotropic colloids have recently attracted growing interest in the soft matter community. ${ }^{1,2}$ Their phase behavior and selfassembly have been investigated theoretically and experimentally for rod-like, ${ }^{2-5}$ ellipsoidal, ${ }^{6-12}$ dumbbell-shaped, ${ }^{13-16}$ bowl-shaped, ${ }^{17-21}$ cubic, ${ }^{9,22}$ and polyhedral particles. ${ }^{23,24}$ However, despite the increasing number of approaches for synthesizing well-defined non-spherical colloids with a large range of particle shapes in the last decade, attempts to synthesize responsive anisotropic particles are still sparse.

Microgel particles have received considerable attention over the years owing to their tunable and responsive nature. ${ }^{25}$ In particular, crosslinked poly( $N$-isopropylacrylamide) (PNIPAM) microgels have been established as a versatile model system where the overall size, softness and hydrophobicity of the resulting particles can be controlled through a temperatureinduced volume phase transition. Thus, their conformation, size, effective volume fraction and interaction potential can be externally controlled and easily adjusted from repulsive to attractive. $^{26-29}$ They exhibit a rich phase behavior ${ }^{26}$ and have been proven to be ideal building blocks for complex selfassembly. ${ }^{30-32}$ Stimuli-responsive microgels have found a broad range of potential applications, such as switchable stabilizers for emulsions at oil/water interfaces ${ }^{33-38}$ and lipid membranes ${ }^{39}$ and also as cell substrates ${ }^{40}$ where the

Division of Physical Chemistry, Department of Chemistry, Lund University,

22100 Lund, Sweden. E-mail: jerome.crassous@fkem1.lu.se

$\dagger$ Electronic supplementary information (ESI) available. See DOI: 10.1039/ c5nr03827h detachment of adsorbed cells can be controlled with the temperature.

So far, anisotropic hybrid microgels have been prepared by using anisotropic particles as seeds for the later synthesis of a thermoresponsive shell. Viruses ${ }^{41}$ Janus dumbbells ${ }^{42}$ or hematite and maghemite spindles ${ }^{43-45}$ are some of the templates reported in the literature. Recently we have shown that polystyrene-poly( $N$-isopropylmethacrylamide) (PS-PNIPMAM) composite microgels can be processed into ellipsoidal particles with adjustable aspect ratios, which greatly enlarges the scope of such particles as it combines an anisotropic design and the functionality of the shell. ${ }^{46,47}$ At the same time, the design of non-spherical colloids makes them interesting candidates for self-assembly processes. Ideally, the key requirement towards complex assembled structures with desirable properties is accurate engineering of colloidal particles. In order to qualify as suitable building blocks with anisotropy in shape and interactions, they need to be highly monodisperse in size and highly uniform in anisotropy. This was demonstrated in our recent study of the dipolar self-assembly of ellipsoidal shaped composite microgels in an alternating field, where for certain aspect ratios the particles were found to self-assemble in well-defined tubular structures. ${ }^{47}$

Herein, we now report that core-shell composite microgels consisting of a PS core and a crosslinked PNIPMAM shell can be nanoengineered into various shapes such as ellipsoidal, faceted and bowl-like. The colloidal particles were prepared by different methods where the initial spherical core-shell microgels were post-processed and reshaped by extending the different procedures usually applied to post-process either spherical polystyrene (PS) or poly(methylmethacrylate) (PMMA) 
particles. ${ }^{48-51}$ A schematic diagram summarizing the different procedures employed is displayed in Fig. 1. The spherical composite microgels were post-processed by either uniaxial mechanical deformation (Fig. 1A), by inducing a localized temperature-controlled phase separation within the PS core swollen by dodecane in a methanol-water mixture (Fig. 1B) or by chemical deformation involving a series of consecutive phase transformations where we use a suitable solvent to swell the core, freeze it in liquid nitrogen and control its extraction at ambient pressure and sub-zero temperature (Fig. 1C).

The reshaping of the microgel particles was confirmed by various techniques, and its effect on their packing properties at high volume fractions was investigated. The thermo-responsiveness of the engineered particles is maintained as revealed by their swelling behavior measured by dynamic light scatter-

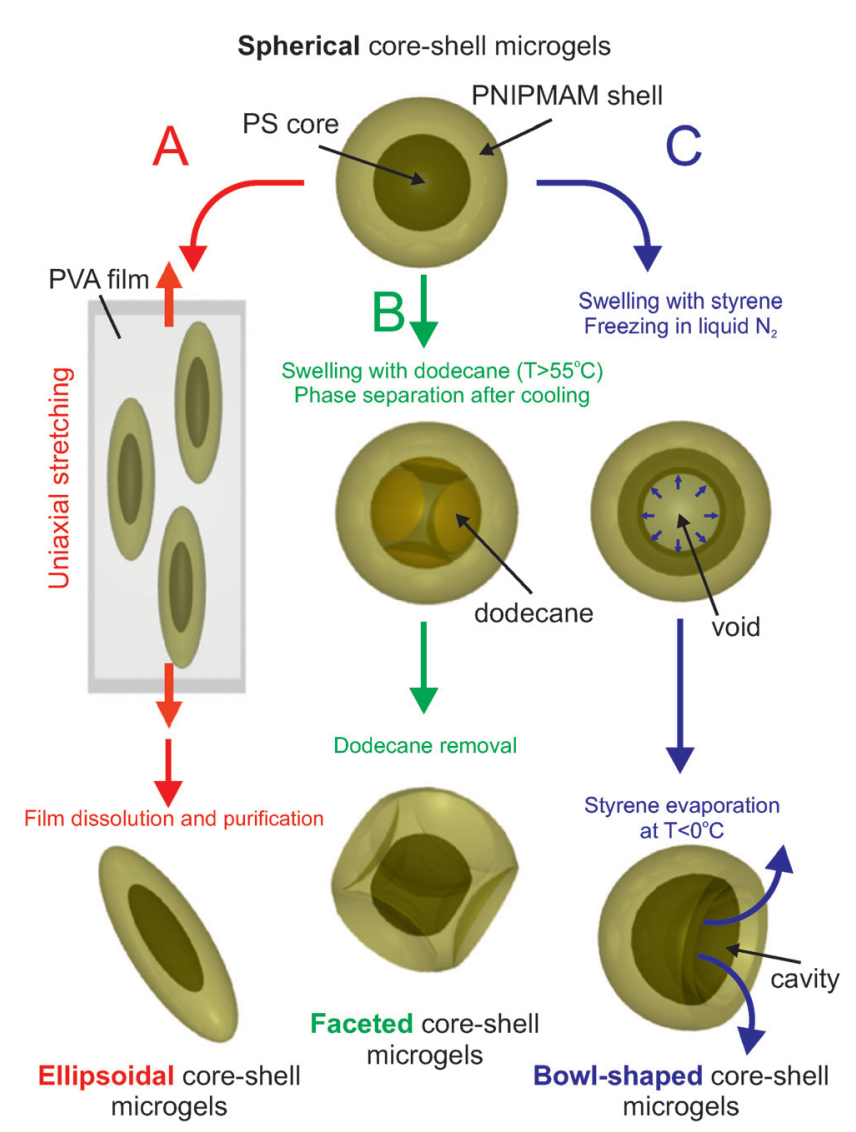

Fig. 1 Schematic illustration of the post-fabrication strategies employed to engineer anisotropic colloids from composite spherical PS-PNIPMAM core-shell microgels. (A) Ellipsoidal particles prepared through uniform deformation of spherical core-shell microgels embedded in PVA films. By this technique a variety of ellipsoid particles are accessible by controlling the deformation ratio. (B) Faceted particles prepared by a temperature-controlled swelling/deswelling of the seed particles in the presence of dodecane droplets in a water/methanol mixture. (C) Bowl-shaped particles formed by swelling of the seed particles with a styrene monomer that are then frozen in liquid nitrogen in order to drive the void formation. Subsequently, the swelling agent was removed by evaporation, allowing macroscopic deformation of the particles and the formation of an aperture. ing (DLS). The dimensions and therefore the effective volume fraction $\phi_{\text {eff }}$ of the dispersed particles can be finely adjusted with $T$ as demonstrated for a dense bowl-shaped dispersion. In addition, the overall nature of their interaction potential can be set from repulsive to attractive by varying the temperature and ionic strength. This is illustrated with the thermo-reversible coagulation of the particles at high ionic strength above their volume phase transition temperature $\left(T_{\mathrm{VPT}}\right)$. Finally, directed self-assembly of the bowl-shaped particles subjected to an alternating electric field is discussed and highlights the potential of using an induced dipolar interaction as an additional control parameter in complex self-assembly processes with anisotropic particles.

\section{Methods}

\section{Materials}

$N$-Isopropylmethacrylamide (NIPMAM; 97\%, Aldrich), $N, N^{\prime}-$ methylenebisacrylamide (BIS; 99\%, Fluka) monomers, sodium dodecyl sulfate (SDS; 99\%, Fluka), potassium peroxodisulfate (KPS; 99\%, Fluka), and polyvinyl alcohol (PVA; 89-98 kDa; 99\%; Aldrich) were used as received. Styrene monomers (BASF) were purified on an $\mathrm{Al}_{2} \mathrm{O}_{3}$ column prior to use. Water was purified using reverse osmosis (MilliRO; Millipore) and ion exchange (MilliQ; Millipore).

\section{Transmission electron microscopy (TEM)}

TEM micrographs were obtained using a Philips CM100Biotwin microscope operating at $80 \mathrm{kV}$. All samples were prepared by placing $4 \mu \mathrm{L}$ droplets of $1 \mathrm{wt} \%$ dispersions onto 300 mesh carbon-coated copper grids at room temperature. After a few seconds excess solution was removed by blotting with filter paper.

\section{Scanning force microscopy (SFM)}

SFM experiments were performed using a Dimension 3100M microscope (Veeco Instruments) equipped with Nanoscope software operated in the tapping mode at room temperature in air. The samples were prepared by spin-coating, at $2000 \mathrm{rpm}$, $0.1 \mathrm{wt} \%$ dispersions onto freshly cleaned silicon wafers. The spring constant of the silicon cantilevers was in the range of 35-92 $\mathrm{N} \mathrm{m}^{-1}$. Scan rates between 0.5 and $1.0 \mathrm{~Hz}$ were used.

\section{Scanning electron microscopy (SEM)}

Scanning electron microscopy (SEM) images of gold sputtered samples were recorded with a FEI Phenom desktop SEM. A drop of the colloidal microgel solution (0.1 wt\%) was deposited and dried on the SEM stubs previously gold sputtered to minimize charging under the fixed conditions before examination.

\section{Confocal laser scanning microscopy (CLSM)}

The confocal micrographs were recorded on a Leica SP5 confocal laser-scanning microscope (CLSM) operated in the inverted mode (D6000I), using a 100×/1.4 NA immersion objective. The 
samples were monitored in situ at different temperatures and an environmental system was employed to ensure the temperature control with an accuracy of $0.2{ }^{\circ} \mathrm{C}$. The suspensions $(8 \mu \mathrm{L})$ were hermetically sealed between two cover glasses separated by a $120 \mu \mathrm{m}$ spacer with a $51 \mathrm{~mm}$ aperture (SecureSeal Imaging). A $543 \mathrm{~nm}$ HeNe laser was used to excite the red fluorescence of rhodamine B (543 nm).

Two different electrode geometries were used for the experiments with an AC electric field. In the first case, the samples were contained between a non-conductive cover slide and a cover slide coated with indium tin oxide (ITO) (SPI Supplies, Structure Probe Inc., USA; 30-60 $\Omega, 18 \times 18 \mathrm{~mm}^{2}$ ) where a $1000 \mu \mathrm{m}$ sample gap was etched out, which ensures a homogeneous field within the gap where CLSM experiments were performed. Conductive tapes were then used to connect the ITO-coated cover slips to the electrodes of the power supply. A sinusoidal electric field $(160 \mathrm{~V}, 160 \mathrm{kHz})$ was applied parallel to the interface, along the $x y$-direction parallel to the image plane, and all the observations were made in the $x y$-image plane. In the second setup the microgel suspension was contained between the two conductive sides of ITO-coated microscope cover slips. An AC field of $20 \mathrm{~V}$ operating at a frequency of $160 \mathrm{kHz}$ was applied to the glass sandwich, normal to the plane. This results in a spatially uniform electric field in the gap between the electrodes. In the current study, we worked at a constant temperature of $20{ }^{\circ} \mathrm{C}$. $3 \mathrm{D}$ reconstructions of the string-like aggregates were realized using the program ImageSurfer. $^{52}$

\section{Dynamic light scattering (DLS)}

DLS experiments were carried out using a light scattering goniometer instrument from LS Instruments equipped with a He-Ne laser $(\lambda=632.8 \mathrm{~nm})$. The temperature was controlled with an accuracy of $0.1{ }^{\circ} \mathrm{C}$. The samples were highly diluted to $0.01 \mathrm{wt} \%$, thus preventing interaction effects and multiple scattering. The DLS measurements were performed at scattering angles of 45,60 and $75^{\circ}$ for temperatures between $20{ }^{\circ} \mathrm{C}$ and $50{ }^{\circ} \mathrm{C}$. Each sample was allowed to equilibrate at the desired temperature for $30 \mathrm{~min}$ before data collection. An apparent hydrodynamic radius $R_{\mathrm{H}}$ was determined from the translational diffusion coefficient, $D_{\mathrm{T}}$, via the Stokes-Einstein relation $D_{\mathrm{T}}=k_{\mathrm{B}} T / 6 \pi \eta R_{\mathrm{H}}$, with $k_{\mathrm{B}}$ being the Boltzmann constant, $T$ the temperature in Kelvin, and $\eta$ the solvent viscosity. The evolution of $D_{\mathrm{T}}$ was determined from the linear dependence of the decay rate $\Gamma v s$. the square of the scattering vector $q^{2}\left(\Gamma=D_{\mathrm{T}} q^{2}\right)$ derived from a first order cumulant analysis of the field autocorrelation function.

\section{Results and discussion}

\section{Spherical core-shell microgels}

The first step towards the design of anisotropic responsive colloids involves the synthesis of the PS-PNIPMAM core-shell particles via a two-step reaction. The core particles were obtained by emulsion polymerization and further used in a second step as seeds for the radical polymerization of the crosslinked shells (details of the synthesis can be found in ref. 46) $\$$ The shell can be fluorescently labeled either by adsorption of the water soluble rhodamine $\mathrm{B}$ dye ${ }^{46}$ or by adding a small amount of the polymerizable rhodamine dye during the synthesis of the shell. ${ }^{47}$ The initial characterization of the non-fluorescent particles was carried out by TEM, SEM and SFM as shown in Fig. 2, which illustrates their sphericity and monodispersity. TEM of the microgels confirms the core-shell structure, where the presence of the shell is indicated by the film formation of close packed particles. In addition, TEM reveals an increase of the radius from $267 \pm 20 \mathrm{~nm}$ to $346 \pm 20 \mathrm{~nm}$ after the shell synthesis. Here, we can assume that these values correspond to a completely collapsed state of the microgels as a result of the drying process required for the TEM sample preparation. Both systems are monodisperse with an average polydispersity below $8 \%$ for the core and $6 \%$ for the core-shell microgel. The core-shell structure is also visible in the two-dimensional (2D) CLSM image as depicted in Fig. 2D of a concentrated (22 wt\%) dispersion after labelling the shell through the adsorption of the water soluble rhodamine B dye. The particles were imaged in the $x y$-plane at the interface between the cover glass and the liquid. As all particles are located in the same image plane, the core-shell character can be easily evidenced in the form of a dark core with a swollen fluorescent corona. Moreover, the local ordering into a hexagonal close packing can also be

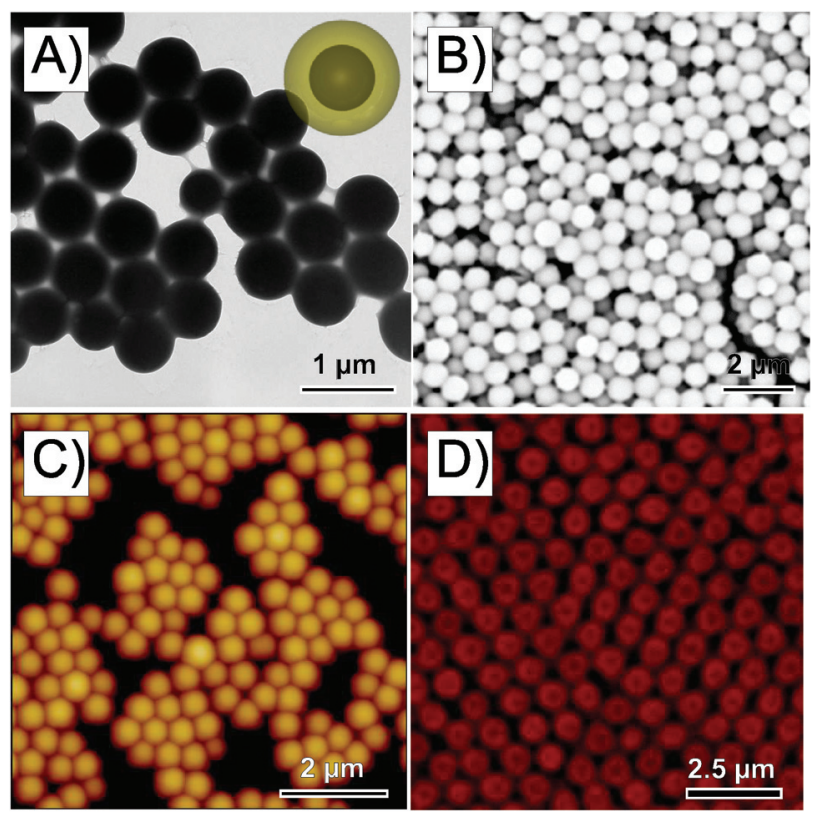

Fig. 2 Spherical particles: PS-PNIPMAM core-shell microgels imaged in the dried state by TEM (A), SEM (B), SFM (C) and in dispersion by CLSM (D)

$\ddagger$ There is an error in ref. 46 for the core synthesis. "NIPAM and SDS were dissolved in $50 \mathrm{~mL}$ pure water" should read "NIPAM and SDS were dissolved in $178 \mathrm{~mL}$ pure water". 
observed. The average particle radius $R \approx 470 \mathrm{~nm}$ was determined from the half distance between the centers of the particles in the crystalline area, in good agreement with the value for the average hydrodynamic radius $R_{\mathrm{H}}=479 \mathrm{~nm}$ obtained from DLS (see Fig. 7).

\section{Ellipsoidal core-shell microgels}

Ellipsoidal particles can be obtained following the approach described by Keville et $a l^{48}$ for poly(methyl methacrylate) particles and further extended to polystyrene particles by Ho et $a .^{49}$ Particles are embedded into a high molecular weight PVA film and then heated above the glass transition temperature, $T_{\mathrm{g}}$, of the polystyrene core. After the uniaxial stretching of the films, the particles adopt an ellipsoidal shape which they retain after cooling below $T_{\mathrm{g}}$. The reshaped particles can then be easily recovered after dissolution of the film. A large variety of complex and defined geometries can be accessed, if one applies a multi-directional stretching of the film and different thermal pathways as shown by Champion et al. ${ }^{53}$ These polymeric prolates were previously investigated extensively in several studies with respect to their dynamics in bulk ${ }^{54,55}$ and at an interface, ${ }^{56}$ their organization in an electric field ${ }^{47,57,58}$ and at high volume fractions, ${ }^{7,10}$ and their application as pickering agents. ${ }^{59}$ We have recently shown that this method can be extended to composite core-shell microgels, where the stretching conditions determine the aspect ratio of the core, while the overall aspect ratio can be further tuned through the thermoresponsive shell. ${ }^{46}$ Following the procedure described in our previous study, ${ }^{46}$ the core-shell particles are embedded into a $100000 \mathrm{~g} \mathrm{~mol}^{-1}$ PVA film. The films are maintained in an oven at a temperature of $180{ }^{\circ} \mathrm{C}$, higher than the $T_{\mathrm{g}}$ of both polymers, for a few minutes and then stretched at different draw ratios. After cooling down to room temperature, the particles can then be recovered by dissolution of the films in hot water and purified by repeated centrifugation. This allows us to tune the aspect ratio up to factor of about 6.5 for a draw ratio reaching $225 \%$. Fig. 3 shows as an example the resulting particles obtained for a draw ratio of $75 \%$.

The thus obtained ellipsoids maintain their temperature responsiveness as illustrated in Fig. 7. For the sample shown in Fig. 3, the average long $(L)$ and short $(d)$ axes were determined from a TEM analysis of more than 100 particles. Values of $L=1672 \pm 258 \mathrm{~nm}$ and $d=472 \pm 57 \mathrm{~nm}$, respectively, were found, resulting in an average aspect ratio of $3.62 \pm 0.86$. The larger polydispersity of the ellipsoids when compared to the initial core-shell spheres is primarily due to the imperfect control of the mechanical stretching process in the oven. An improved procedure that uses a better regulated stretching device where the film is homogeneously heated in a silicon oil bath has allowed us to significantly decrease the polydispersity of the particles. ${ }^{47}$ The average dimensions obtained from statistical CLSM image analysis of the particles adsorbed at the glass/water interface at $20^{\circ} \mathrm{C}$ are $L=1839 \pm 208 \mathrm{~nm}$ and $d=$ $668 \pm 68 \mathrm{~nm}$, which results in an average axial ratio of $2.79 \pm$

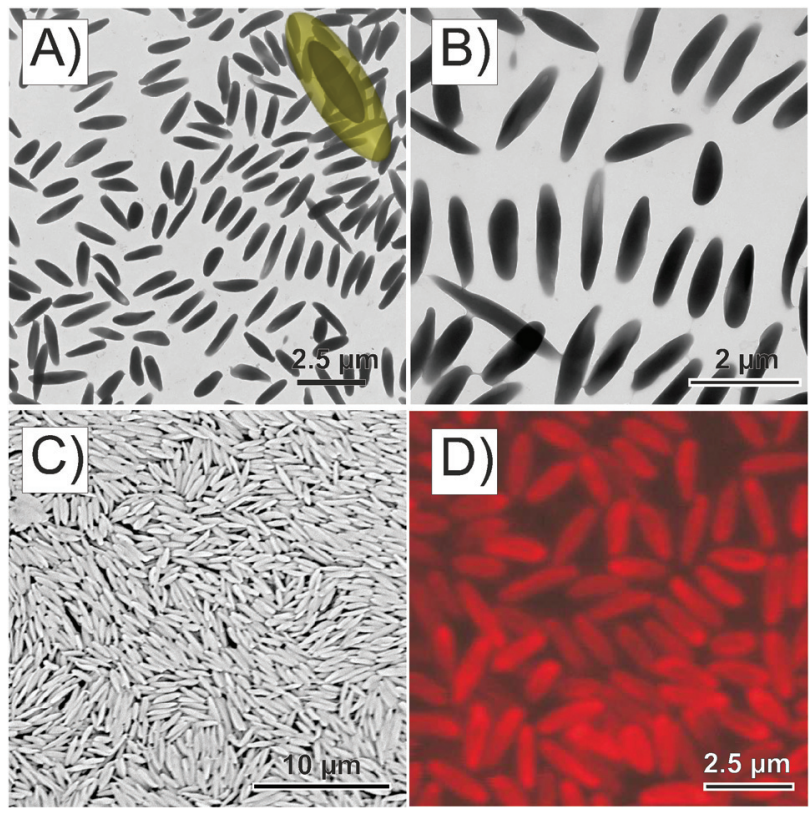

Fig. 3 Ellipsoidal PS-PNIPMAM core-shell particles obtained from spherical core-shell particles following the method described by Ho et al.: ${ }^{49}$ TEM (A, B), SEM (C) and CLSM (D) images.

0.475. The difference between the TEM and the CLSM results is caused by the fact that the TEM measurement provides dimensions for a fully collapsed state of the shell, whereas CLSM yields the values in suspension, i.e. with a fully swollen PNIPAM shell. This indicates that the responsive nature of the shell allows us to change not only the overall size but also the shape (axial ratio) in a controlled and completely reversible manner.

\section{Faceted core-shell microgels}

Faceted particles are another interesting class of anisotropic colloids. Here we start from the previously reported method to engineer dimple PS lattices from simple spherical particles by taking advantage of the spinodal decomposition between the polystyrene and dodecane in water-methanol mixtures. ${ }^{50}$ The polystyrene and dodecane become miscible at high temperatures where the dodecane swells the polystyrene. When the dispersion is cooled down, the two components phase separate and the dodecane is expelled from the particles in the form of a droplet creating a dimple at the PS/dodecane interface (determined by the minimization of the interfacial free energy). By controlling the stirring rates and the initial solvent composition, the dimple size could be controlled and therefore the shape of the resulting particles could be tuned from slightly truncated spheres up to hemispheres. The same procedure was further applied to create PS particles where the facets were specifically functionalized to direct the spontaneous association of the particles into dumbbells or trimers through hydrophobic interactions. ${ }^{60}$ 
We subsequently adapted the procedure reported by Tanaka et al. ${ }^{50}$ to core-shell composite microgels. In a typical preparation, a $0.6 \mathrm{~mL}$ microgel suspension (2.42 wt \%) was heated under stirring at $400 \mathrm{rpm}$ to $70{ }^{\circ} \mathrm{C}(1 \mathrm{~h})$ in a watermethanol mixture (16/84), and in the presence of $0.1 \mathrm{~g}$ dodecane. The phase separation between the polystyrene and the dodecane is induced by slowly cooling down the mixture up to $25^{\circ} \mathrm{C}$ at a rate of about $1{ }^{\circ} \mathrm{C} \min ^{-1}$ under continuous stirring at $400 \mathrm{rpm}$ for $1 \mathrm{~h}$. The suspension was then purified by repeated centrifugation (3 times at $10000 \mathrm{rpm}$ ) and redispersed in water. Fig. 4 illustrates the resulting multi-faceted particles. Another interesting aspect, visible from the different microscopy techniques in the dried state, is the presence of sharp edges at the surface of the particles (see Fig. $4 \mathrm{~A}-\mathrm{C}$ ). The formation of well defined facets can be attributed to local confinement effects at the core-shell interface during the spinodal decomposition. As previous studies demonstrated, ${ }^{61}$ the microgel shell is not fully attached to the polystyrene core but rather exhibits some buckling at the core-shell interface, and is expected to be at the origin of the resulting morphology. The processed particles were also imaged in dispersion using CLSM at $20^{\circ} \mathrm{C}$ as displayed in Fig. 4D. The swollen shell can be clearly distinguished from the CLSM images, and the particles still appear faceted but with much smoother edges than in the dried state. Thus, the extension of this approach to core-shell particles enlarges the field of accessible morphologies for microgels. Moreover, similarly to the ellipsoidal particles, where the degree of anisotropy can be altered with the temperature, we expect to be able to control the roundness ( $c f$. angularity) of the particles, and thus their aeolotropic interaction potential by changing the conformation of the microgel shell.

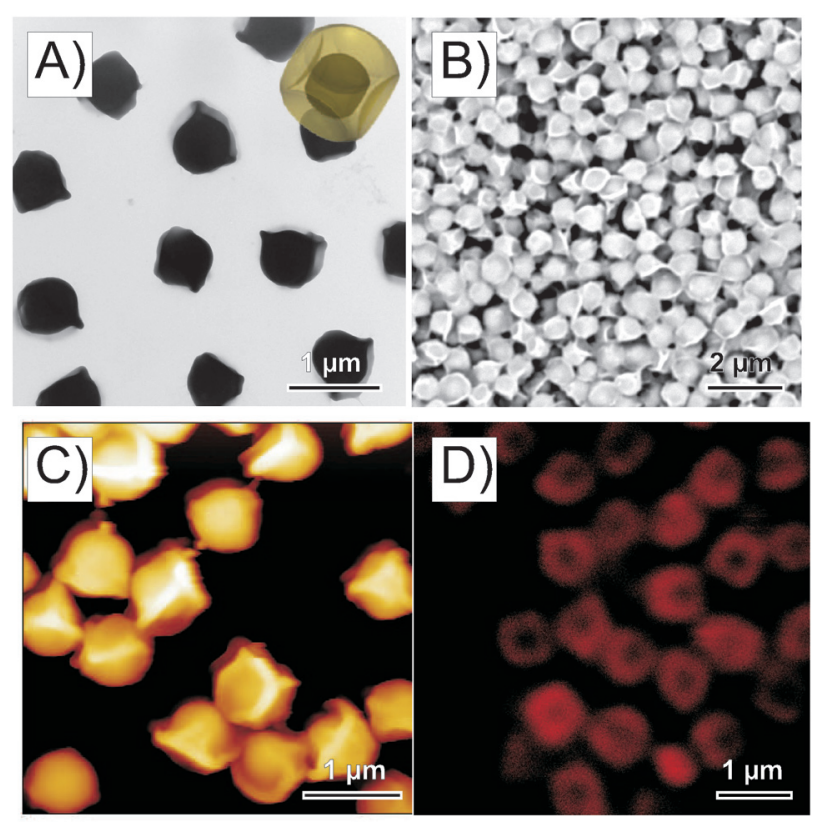

Fig. 4 Images of faceted PS-PNIPMAM core-shell particles: TEM (A), SEM (B), SFM (C) and CLSM (D).

\section{Bowl-shaped core-shell microgels}

The fabrication of hollow or bowl-shaped PS or PMMA particles with controllable holes in their surfaces has been reported by Im et al. ${ }^{51}$ The particles were first swollen with a good solvent (either styrene or toluene), and then quickly frozen in liquid nitrogen $\left(-196^{\circ} \mathrm{C}\right)$. As the solvent (toluene or styrene) is solidified, a void is created at the center of the particles. In the next step, the frozen sample is slowly warmed up to let the swollen agent evaporate. The evaporation process was carried out below $0{ }^{\circ} \mathrm{C}$ under vacuum or at ambient pressure. Due to the presence of an evaporation flux, a hole appears in the shell of each hollow particle. By controlling the evaporation conditions (pressure and temperature), these authors have demonstrated that the size of the void and aperture can be finely adjusted. We refer to this work for more details of the transformation mechanism. Interestingly, the existence of an aperture at the surface of the particles could be employed to encapsulate functional species such as proteins or magnetic nanoparticles.

Here, a similar approach is applied to the spherical coreshell microgel particles. In the first step, purified styrene $(0.2 \mathrm{~mL})$ was used as a swelling agent and added to $4 \mathrm{~mL}$ $1.2 \mathrm{wt} \%$ core-shell latex dispersion in MilliQ water. The mixture was then homogenized for $1 \mathrm{~h}$ under continuous stirring at $300 \mathrm{rpm}$. Next, the dispersion was added dropwise to a $2 \mathrm{~L}$ beaker filled with liquid nitrogen. After the addition of particles, the suspension was kept in the freezer $\left(-10^{\circ} \mathrm{C}\right)$ for $48 \mathrm{~h}$. In the last step, the frozen sample was brought back to room temperature and maintained here until the drops became liquid again. Subsequently, we added $10 \mathrm{~mL}$ of water to fully recover the system. The final product was then purified by repeated centrifugation and washed several times with deionized water.

Fig. 5 shows typical examples of the prepared bowl-shaped core-shell microgels by TEM, SEM and CLSM imaging. The TEM analysis (see Fig. 5A and B) confirms that all the particles exhibit a cavity and are rather monodisperse in size and shape. The transformation into bowl shape is also visible from particles pointing perpendicularly to the image plane that exhibit a lighter area in their center, in contrast to the original spherical particles shown in Fig. 1A. Their bowl-shaped character is also clearly visible from the SEM micrograph shown in Fig. 5C. An overall radius of $391 \pm 26 \mathrm{~nm}$ was derived from TEM, which is larger than the radius of the initial spherical particles. The average wall thickness of $134 \pm 28 \mathrm{~nm}$ was also estimated from TEM by analyzing the particles pointing perpendicularly to the image plane, and an average aperture radius of $310 \pm 35 \mathrm{~nm}$ was obtained from the SEM micrographs. The bowl-shaped microgels were imaged in dispersion by CLSM at the water/ glass interface (see Fig. 5D and E). Here post-processed covalently labeled fluorescent particles were investigated. These particles have a similar shape to the unlabeled ones as confirmed by TEM and CLSM. CLSM images performed at $20^{\circ} \mathrm{C}$ indicate that the fluorescent PNIPMAM shell is surrounding the entire PS core. The core-shell character is reflected by the 

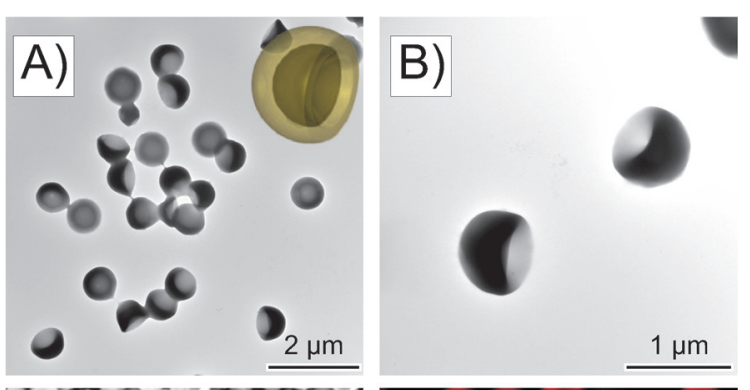

E)
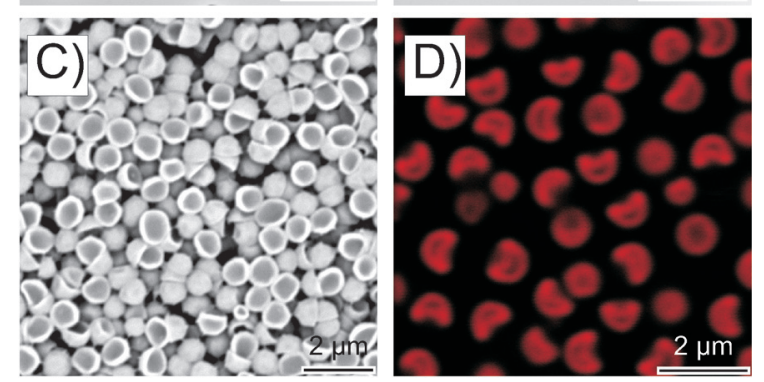

Fig. 5 Images of bowl-shaped PS-PNIPMAM composite microgels: TEM (A, B), SEM (C) and CLSM (D). 3D CLSM projection of the bowl-shaped particles which was reconstructed from confocal $z$-stack images on a volume of $2.93 \times 2.93 \times 3.56 \mu \mathrm{m}^{3}(\mathrm{E})$.

presence of a dark area in the center of the particles. The statistical analysis of the CLSM micrographs yields an overall radius, $R_{\mathrm{T}}=497 \pm 16 \mathrm{~nm}$, which is larger than the one determined from TEM and demonstrates that the particles are swollen. In addition the bowl-shaped conformation is maintained in the swollen state, which is supported by the 3D reconstruction presented in Fig. 5E. This suggests that the particle geometry can be further adjusted with the temperature by controlling the swelling of the shell.

\section{Effect of anisotropy on the packing of particles in dense} suspensions

The different anisotropic particles described above were then used to investigate the effect of shape on the packing at maximum density. The samples were first centrifuged for $10 \mathrm{~min}$ at $10000 \mathrm{rpm}$, and then the supernatant was removed and the dispersions were sonicated for $30 \mathrm{~min}$ in an ice bath. Fig. 6 shows CLSM micrographs taken at about $3 \mu \mathrm{m}$ from the cover glass/water interface for the ellipsoidal (A), faceted (B) and bowl-shaped (C) particles after about 2 hours equilibration at $20^{\circ} \mathrm{C}$. Monodisperse ellipsoids with an aspect ratio of 2.79 are expected to first exhibit a nematic phase at intermediate volume fraction followed by a simple monoclinic phase at higher densities. ${ }^{12,62}$ Our ellipsoids, however, only exhibit a local nematic order, similarly to what could be observed from the SEM analysis of a dried dispersion (see Fig. 3C), which is probably due to the relatively high polydispersity of the particles and the possible existence of an intervening glass transition. ${ }^{4,63}$
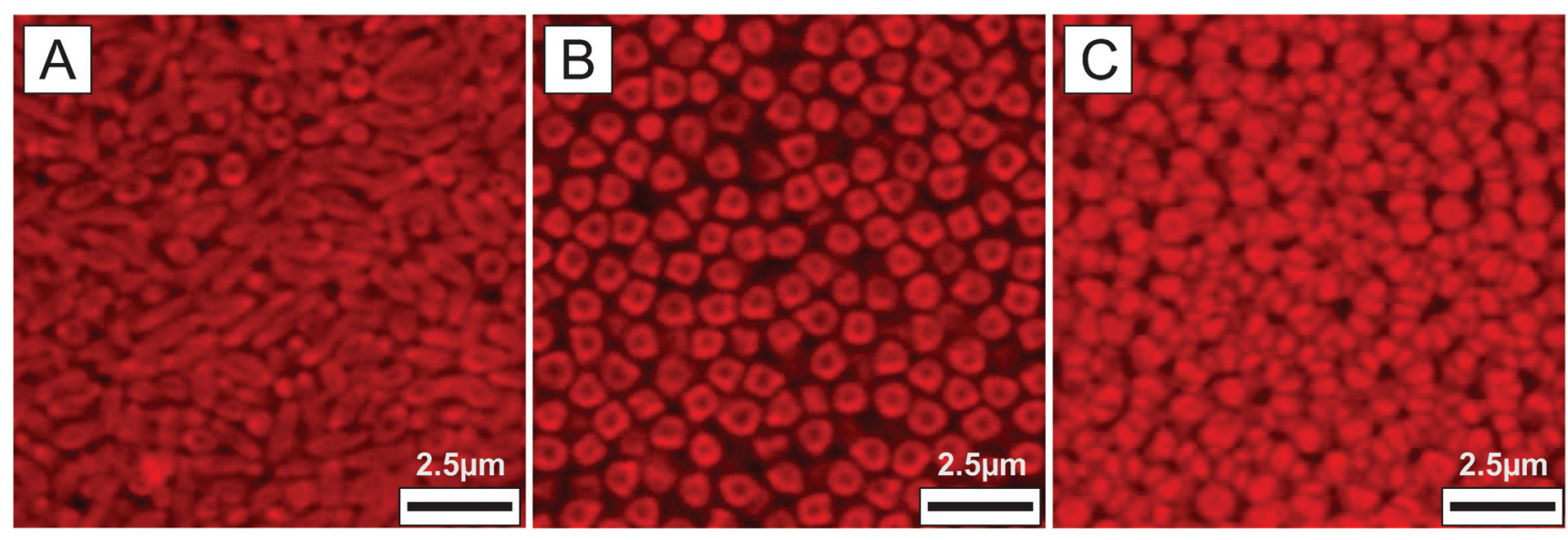

Fig. 6 CLSM micrographs of jammed dispersions of ellipsoidal (A), faceted (B) and bowl-shaped (C) nanoengineered composite microgels imaged at $20^{\circ} \mathrm{C}$ at about $3 \mu \mathrm{m}$ from the cover slide. 
The faceted particles densely pack by maximizing their contact with their neighbours through the alignment of their facets. It is interesting to note that the irregular faceting of the particles leads to the formation of larger voids (or defects) in the jammed dispersion. This observation is similar to the case of granular materials such as sand when the roundness of the grains decreases. This indicates that responsive anisotropic particles where both anisotropy or eccentricity and angularity can be tuned through a combination of initial processing conditions (stretching methods combined with subsequent transformations) and temperature response could be used as interesting model systems for studies of packing and jamming in granular materials. ${ }^{64}$

Dense suspensions of bowl-shaped particles were found to locally stack, thus forming interconnected worm-like structures. This effect is similar to what has been observed in other experimental and simulation studies. ${ }^{17}$ In these previous experiments on hard and widely opened bowl-shaped particles, long stacks of particles were observed after slow sedimentation. At the simulation level, for sufficiently deep bowls a columnar phase is expected to be thermodynamically stable, whereas for less deep bowls a more complex phase diagram was predicted. In comparison, our bowl-shaped composite microgels exhibit weaker and more local order with a high density of junctions. We believe that this can be attributed to the particular particle shape with an aperture that is significantly less deep and with a rounded rim, the softness of the potential, and to the fact that high density is achieved through a centrifugation procedure that may not allow the particles to establish equilibrium properly. We believe that the combination of a responsive shape and a soft potential will require additional theoretical and simulation work to establish the underlying phase behaviour.

\section{Exploring the thermosensitivity of the anisotropic-shaped composite microgels}

In contrast to the previously reported PS or PMMA particles, the crosslinked PNIPMAM shell of our particles allows us to actively change their softness, hydrophobicity and size with temperature. The thermoresponsive behavior of the anisotropic colloids was first investigated using DLS. Fig. 7 displays the evolution of the apparent hydrodynamic radius $R_{\mathrm{H}}$. The DLS data reveal that the thermosensitivity of the spherical seed microgels is maintained when post-processing the particles. Fig. 7 shows that as the temperature is increased, all reshaped colloidal particles exhibit a volume phase transition, VPT, and are well-dispersed. Above $T_{\mathrm{VPT}}$, the microgels undergo a collapse transition and their hydrophobicity is strongly altered. The VPT occurs at $42{ }^{\circ} \mathrm{C}$ for the spherical seed microgels, which shifts to a lower value of $38^{\circ} \mathrm{C}$ for the ellipsoidal particles, and to a higher value of $45{ }^{\circ} \mathrm{C}$ for the faceted particles. The bowl-shaped particles display the VPT at around $42{ }^{\circ} \mathrm{C}$, similar to the spherical seed particles. The shift of the VPT of the ellipsoidal particles to a lower temperature was attributed to the stretching process which may have reduced the swelling of the particles, similar to that observed in a conventional elas-

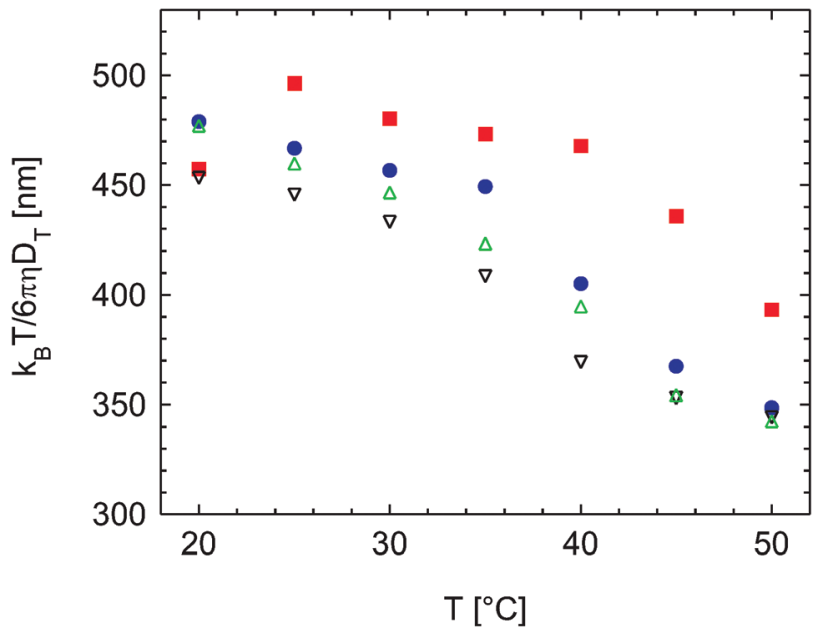

Fig. 7 Apparent hydrodynamic radius $R_{\mathrm{H}}$ of different shaped core-shell microgel particles as determined by DLS: spherical (blue filled circles), faceted (red filled squares), bowled (green up triangles), and ellipsoidal (black down triangles).

tomeric network as discussed in a previous study. ${ }^{46}$ The estimated swelling ratio defined as $\left(R_{\mathrm{H}}\left(20^{\circ} \mathrm{C}\right) / R_{\mathrm{H}}\left(50^{\circ} \mathrm{C}\right)\right)^{3}$ varied from 2.6 for the spherical seed core-shell particles to 1.6 (faceted), 2.3 (ellipsoidal) and 2.7 (bowled), respectively. The volume phase transition behavior of the particles is thermally reversible.

In the following the thermoresponsiveness of the nanoengineered composite microgels is further illustrated by looking at the structural properties of a dense bowl-shaped particle dispersion obtained by centrifugation in pure MilliQ water. The particles were visualized by CLSM; however, the turbidity of the concentrated sample arising from the refractive index mismatch only allows for a clear localization of the particles up to about $10 \mu \mathrm{m}$ from the cover glass. Fig. 8(A, C, E, G, I) show typical micrographs of dispersions at different temperatures, recorded at a sample position after the first adsorbed layer. A particle number density $n_{\mathrm{p}} \approx 1.54 \mu \mathrm{m}^{-3}$ was estimated from the evaluation of three consecutive planes. Using an effective single particle volume of $V_{\mathrm{p}} \approx 0.7678(4 / 3) \pi R_{\mathrm{T}}{ }^{3} \mu \mathrm{m}^{3}$ determined from the CLSM micrographs as described in the ESI (see Fig. S2 $\dagger$ ), we can estimate an effective volume fraction $\phi_{\text {eff }}=$ $n_{\mathrm{p}} V_{\mathrm{p}} \approx 0.61$ at $20^{\circ} \mathrm{C}$.

The 2D pair correlation functions (2D $g(r) s)$ determined at different temperatures (Fig. 8(B, D, F, H, J)) show that the particle positions are strongly correlated. At $20{ }^{\circ} \mathrm{C}$ the particles were found to exhibit significant liquid-like order with a local hexagonal arrangement. The amplitude of the first peak in the $2 \mathrm{D} g(r)$ was $g_{\mathrm{m}}=3.02$ and its position $r_{\mathrm{m}}=0.988 \mu \mathrm{m}$ at $20{ }^{\circ} \mathrm{C}$, which is comparable to the hydrodynamic diameter of the particles. Interestingly, the second peak exhibits some splitting similar to what can be observed for dense spherical microgel dispersions in a glassy state. ${ }^{65,66}$ An analysis of their center of mass positions with time shows that the system is indeed in a glassy state where the individual particles can only undergo 

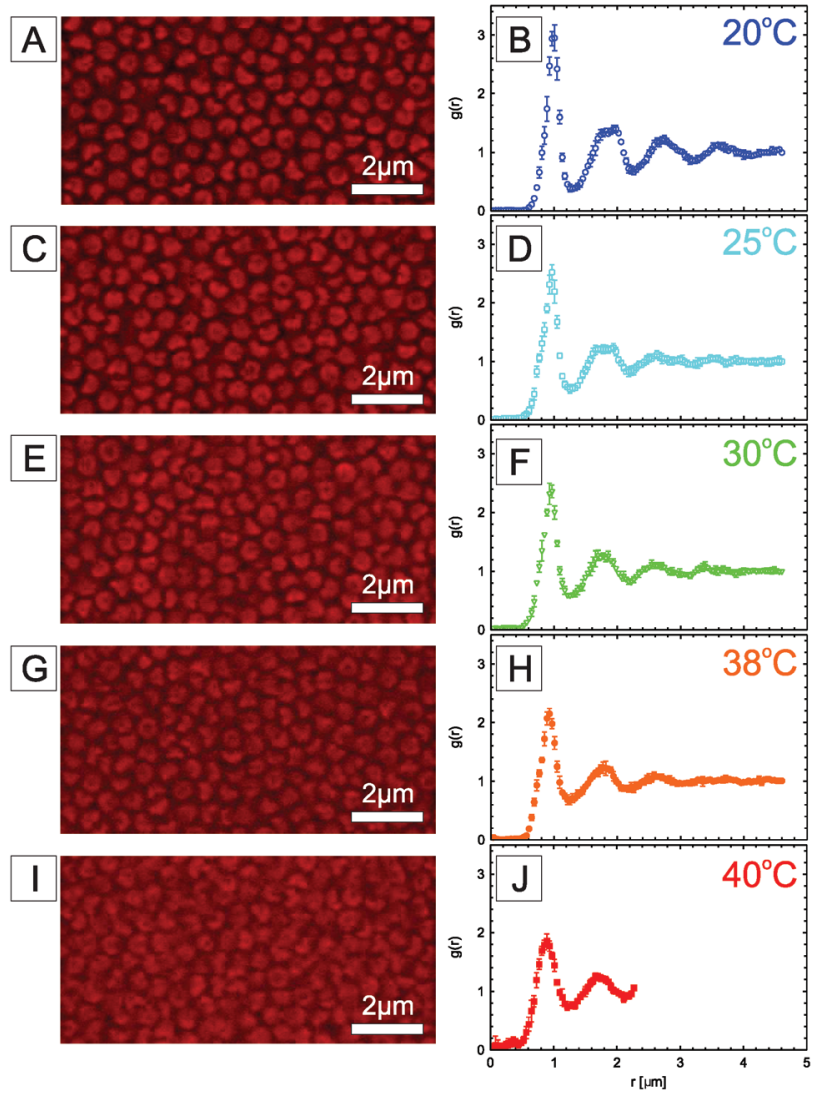

local motion and where their trajectories are confined to cages formed by their neighbours. As shown in Video S1 of the ESI, $\dagger$ the anisotropic particle shape allows us to visualise the rotation of the particles, and we see that individual particles are confined to their cages and maintain a given orientation for some time, but then stochastically flip their orientation.

The dispersion structure shown in Fig. 8A differs from the denser suspension in Fig. 6C, which showed small chains of stacked particles. This is confirmed by a more detailed analysis of the local particle arrangement that was performed for three consecutive stacks as shown in Fig. 9. Here, we first analyse the center of mass positions and the packing density by considering spheres inscribing the individual particles. Fig. 9B illustrates the presence of a large number of overlaps between the circumscribing spheres. This indicates that individual bowl-shaped particles will experience restrictions on their orientation and provides a reason for the absence of continuous rotational motion. The orientation of individual particles is temporarily blocked due to packing constraints and can only change when a stochastic cage rearrangement allows for a flipping motion. However, a closer look at their projected orientation in the image plane defined by the vector normal to the aperture (Fig. 9C) shows that their orientation appears relatively random and no clear orientational correlation typical for stacked chains or liquid crystalline structures exists.

The 2D pair correlation functions determined at different temperatures (Fig. 8(B, D, F, H, J)) directly reflect the temperature-responsive size and shape of the particles. The particles become smaller with increasing temperature (Fig. 7), resulting in a decrease of $g_{\mathrm{m}}$ to 1.86 and of $r_{\mathrm{m}}$ to $0.890 \mu \mathrm{m}$ at $45^{\circ} \mathrm{C}$. From 20 to $38{ }^{\circ} \mathrm{C}$ the dispersions were in a glassy state with an arrested center of mass diffusion where the particles were found to rattle within the cage formed by the other particles. However, at higher temperatures, as the particle size decreases, the local dynamics speeds up and the particles rotate more homogeneously. This demonstrates the potential use of the thermoresponsive composite microgels with a tuneable size and shape to explore the effects of shape anisotropy on the phase diagram and the related local dynamics. In particular, the fact that we can monitor not only the position of the center of mass of the particles but also their orientation and rotational motion provides us with an interesting tool to look at various arrest scenarios where we can distinguish between glass formation and jamming, and determine the onset of orientational correlations.

It is well-known that composite microgel particles can become attractive at higher temperatures if the electrostatic interactions are sufficiently screened. ${ }^{28,29,45}$ Therefore, not only the size but also the interaction potential can be efficiently adjusted with temperature. This is shown for a bowl-shaped particle dispersion prepared at high ionic strength $(0.16 \mathrm{wt} \%$, $0.1 \mathrm{M} \mathrm{KCl}$ ). Below $40{ }^{\circ} \mathrm{C}$ the suspension is stable, but a further increase of the temperature leads to destabilisation of the suspension and to the formation of large branched aggregates. When the temperature is decreased back to below $40{ }^{\circ} \mathrm{C}$ the particles redisperse, confirming the reversibility of the process. The coagulation was monitored during the quenching of the suspension to $50{ }^{\circ} \mathrm{C}$ (see ESI Video $\mathrm{S} 2 \dagger$ ). The particles were found to first form smaller linear aggregates which further interconnect to build up larger branched quickly sedimenting aggregates (see Fig. 10). The particle orientation within the aggregates appears to be random (see Fig. 10). It is interesting to note that at an ionic strength of $10^{-3} \mathrm{M} \mathrm{KCl}$ the particles are stable over the full temperature range. A similar observation could be made with the other nanoengineered composite microgels, thus demonstrating that their interaction potential can be efficiently tuned from repulsive to attractive by properly adjusting both the ionic strength and the temperature.

\section{Field-directed self-assembly}

To further explore the use of the reshaped composite microgels as building blocks for supracolloidal structures, we investigate the field-assisted assembly of the bowl-shaped particles in an alternating (AC) electric field. Previous studies have shown that AC fields can be used to manipulate the assembly of Janus spheres ${ }^{67,68}$ and rods, ${ }^{69}$ ellipsoidal, ${ }^{57}$ spheroidal $^{70}$ 

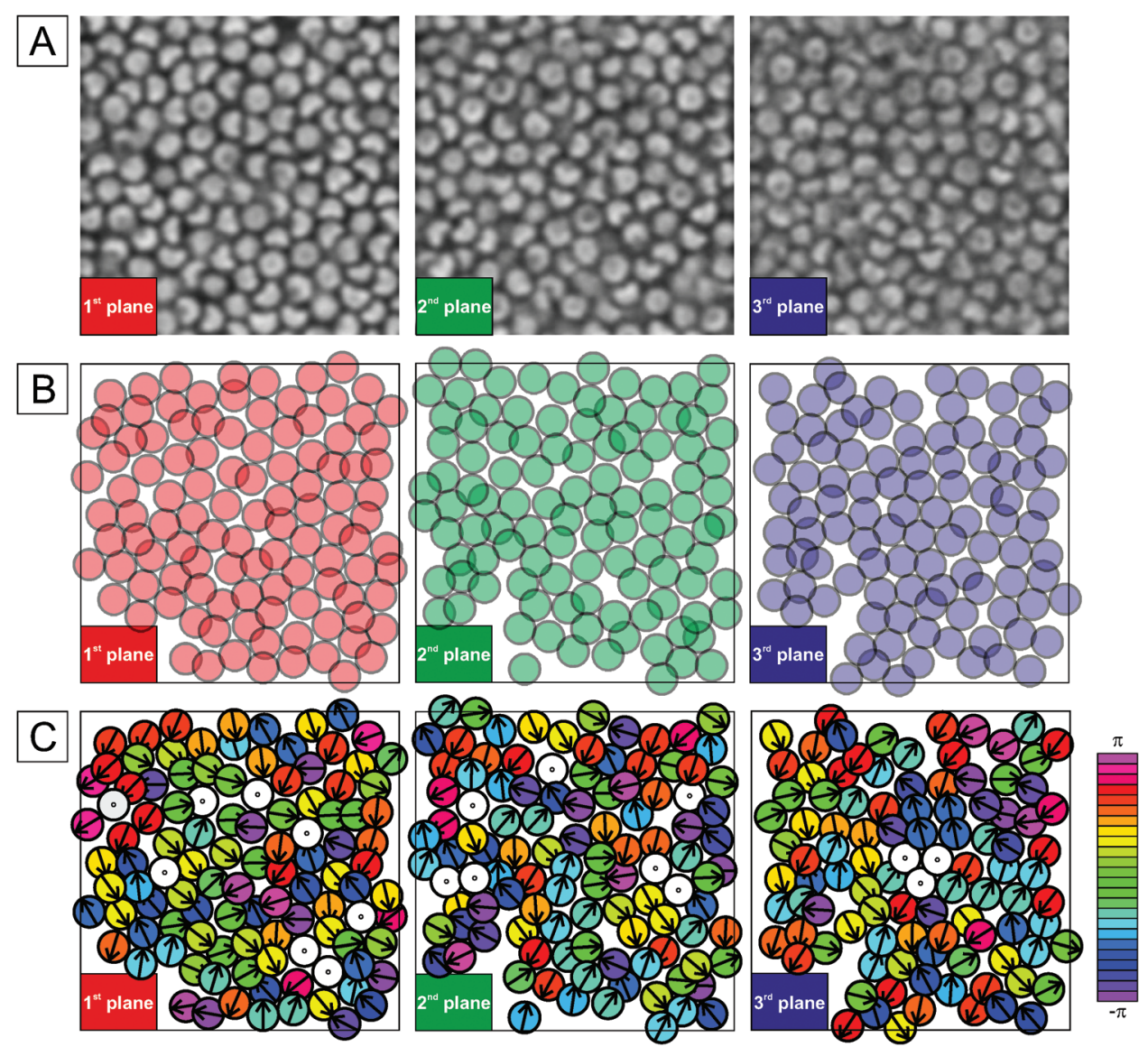

Fig. 9 (A) 2D CLSM micrographs of three consecutive planes in a dense bowl suspension measured at $20^{\circ} \mathrm{C}$ (see Fig. $7 \mathrm{~A}$ ). The first plane corresponds to the first layer in bulk after the adsorbed layer. (B) Manual analysis of the position of individual particles in the focal plane where particles are represented by spheres $(1 \mu \mathrm{m}$ in diameter) inscribing the bowl-shaped composite microgels. (C) Analysis of the projected orientation of the particles in the plane defined by the director normal to the aperture. The orientation is supported by the color code with the white color referring to particles pointing in the $z$-direction.
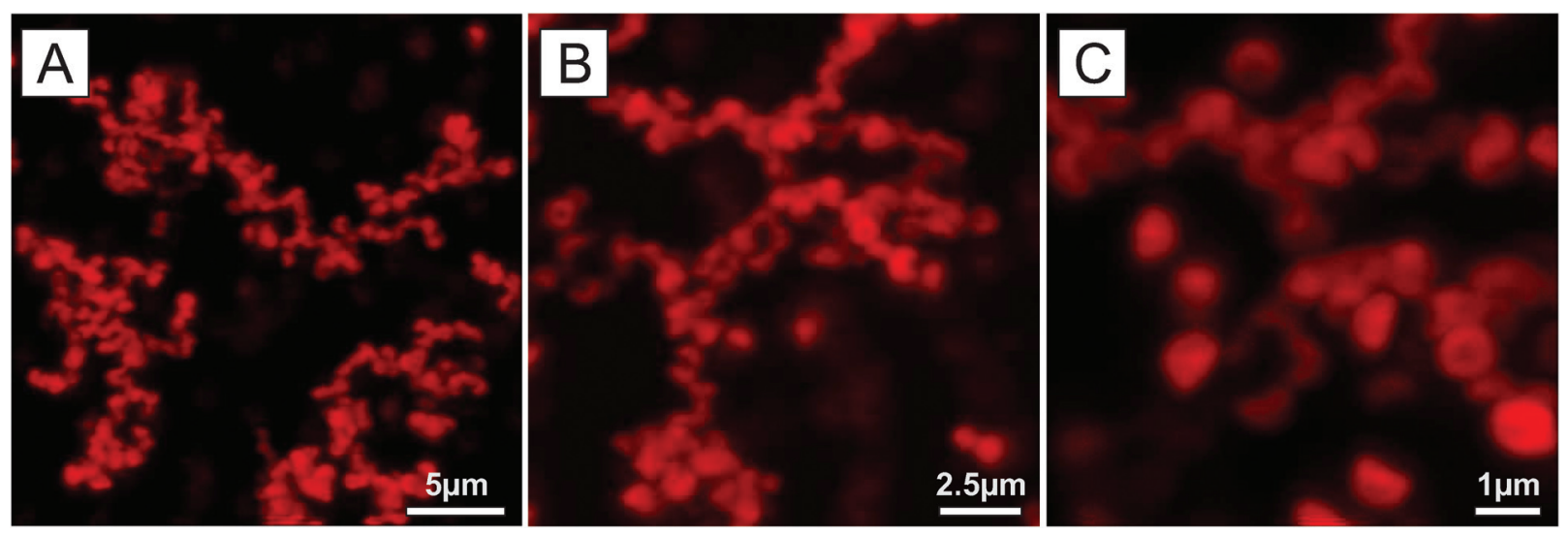

Fig. $10 \mathrm{CLSM}$ micrographs of the fractal aggregates formed after quenching a bowl-shaped composite microgel dispersion (0.16 wt\%, $0.1 \mathrm{M} \mathrm{KCl)}$ from $20{ }^{\circ} \mathrm{C}$ to $50{ }^{\circ} \mathrm{C}$ (see ESI Video $2 \uparrow$ for the aggregation process). The micrographs, obtained from the maximal intensity projection of $2 \mathrm{D}$ images corresponding to a stack volume of $25.83 \times 25.83 \times 8.04 \mu \mathrm{m}^{3}$ (A), $15.50 \times 15.50 \times 7.30 \mu \mathrm{m}^{3}$ (B) and $7.75 \times 7.75 \times 5.18 \mu \mathrm{m}^{3}$ (C), evidence the random orientation of the particles within the aggregates.

and bowl polystyrene particles, ${ }^{71}$ composite dumbbells, ${ }^{72}$ and spherical PNIPAM ionic microgels, ${ }^{73,74}$ respectively. Recently, the AC field-induced self-assembly was investigated for spherical and ellipsoidal composite microgels ${ }^{47}$ and we therefore refer the reader to this work for more details of the polarization mechanism. 
Fig. 11 displays typical structures formed by bowl-shaped composite microgels in a $1 \mathrm{wt} \%$ dispersion in the presence of an $\mathrm{AC}$ electric field at $20^{\circ} \mathrm{C}$. At the beginning of each experiment when the electric field is off, the bowl particles are randomly oriented in a fluid state. Dipolar chains form when the induced dipolar attraction between the particles is sufficiently strong to overcome the thermal energy $k_{\mathrm{B}} T$. The particles in a string have a preferred orientation where the director normal to the aperture is perpendicular to the applied field, and are able to rotate around the string axis (see ESI Video S ${ }^{\dagger}$ and Fig. 11A and B). As the particles are not density matched, the strings were found to quickly sediment and concentrate at the bottom of the preparation where they formed a dense string fluid (see Fig. 11C and ESI Videos S4 and S5 $\dagger$ ). In this state, the particles maintain their ability to rotate and were found to self-organize in the image plane into well-ordered slightly distorted hexagonal arrays. This could be evidenced by the timeaverage Fourier transform analysis of the string fluid imaged in the $x y$-plane (see Fig. 11D). A more detailed analysis including the analysis of the corresponding $2 \mathrm{D} g(r) \mathrm{s}$ is available in the ESI. $\dagger$

The same experiment was repeated, this time with the electric field oriented normal to the imaging plane (along the $z$ direction in the CLSM image-plane). Fig. 11E shows 2D snapshots from image planes $(x y)$ perpendicular to the field direction, obtained at a field strength of $167 \mathrm{kV} \mathrm{m}^{-1}$ and a frequency of $160 \mathrm{kHz}$. Long strings formed that span the full gap between the two electrodes, thus preventing their sedimentation. After a few minutes, we observed a structural transition from a string fluid to a crystal with a body-centered tetragonal (BCT)-like structure, coexisting with tubular aggregates (see Fig. 11E). The ordered colloidal domains were reconstructed from confocal $z$-stack images (Fig. 11F-H and Video $\mathrm{S} 6 \dagger)$. To the best of our knowledge, 3D colloidal crystals or tubes have not been reported for field-assisted assemblies of similar systems such as bowl particles or particles with spherical cavities. ${ }^{19,71}$ The assembly process is fully reversible, i.e., the colloidal dispersion relaxes to its initial fluid-like disordered state when the field is turned off and tubes and crystals reform when the AC electric field is re-applied. The swollen microgel shell is likely responsible for the fast redispersion process as it ensures the steric stabilization of the particles and exhibits much lower van der Waals interactions compared to polystyrene or silica particles. It is interesting to note that the spherical particles were found to arrange in a BCT structure, whereas the more elongated ellipsoidal compo-
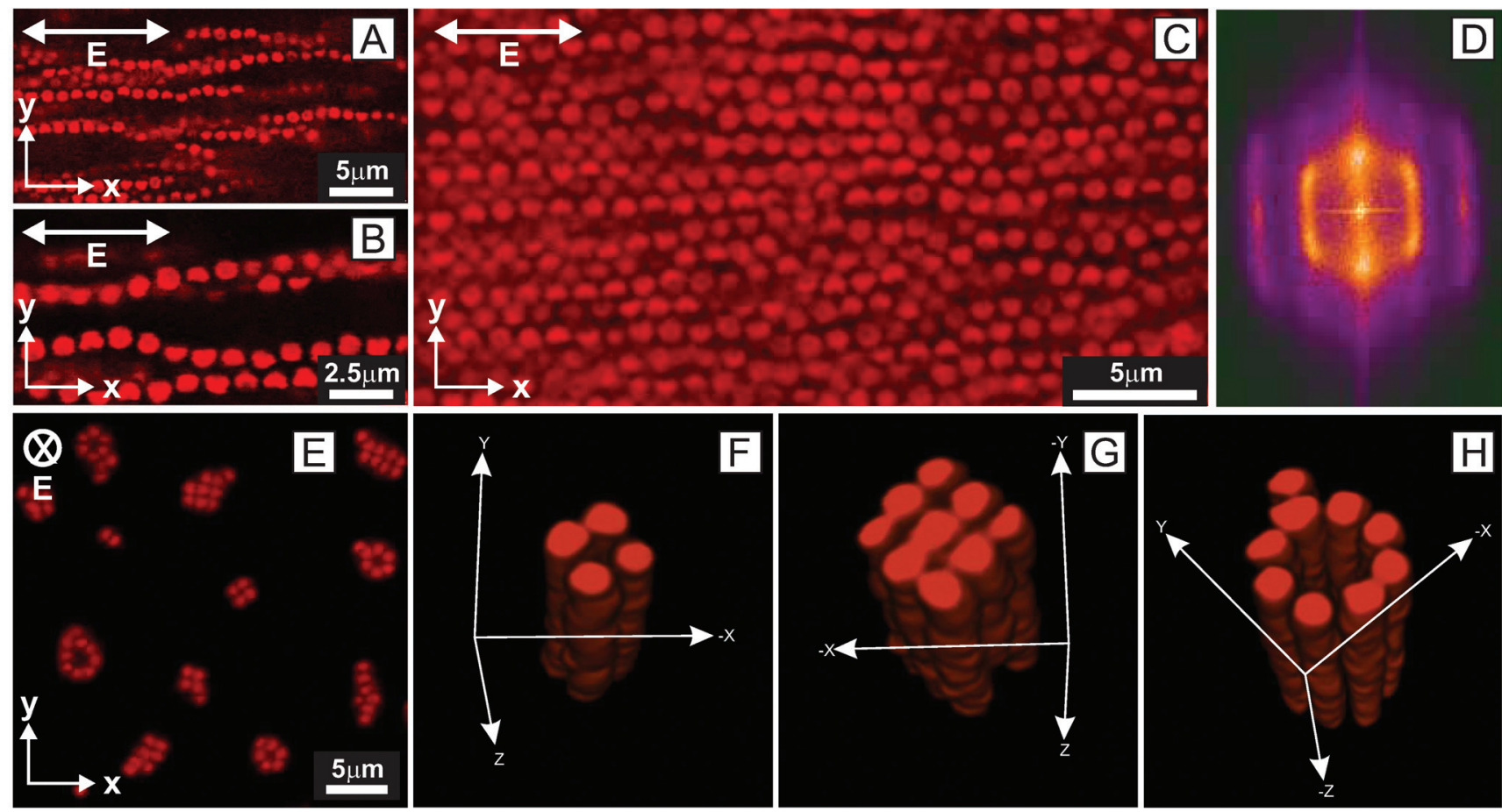

Fig. 11 CLSM micrographs of the self-assembled bowl-shaped composite microgels (1 wt\%) in an AC electric field recorded at $20^{\circ} \mathrm{C}$. (A-D) Experiments performed with the field applied in the $x$-direction at a field strength $E=160 \mathrm{kV} \mathrm{m}^{-1}$ and a frequency $f=100 \mathrm{kHz}$. (A, B) $2 \mathrm{D} \mathrm{micrographs} \mathrm{of}$ the string formation along the field consisting of rotating bowl-shaped particles with their aperture pointing perpendicular to the field (see ESI Video S2 $\dagger$ ). (C) Dense particle string fluid formed at the bottom of the sample after sedimentation of the strings (see ESI Video $3 \uparrow$ ). (D) Time-averaged FFT analysis of the dense string fluid shown in $(C)$ evidencing the organization of the particles in slightly deformed hexagonal arrays. (E-H) Experiments performed with the field applied in the $z$-direction at a field strength $E=167 \mathrm{kV} \mathrm{m}^{-1}$ and a frequency $f=160 \mathrm{kHz}$. (E) $2 \mathrm{D}$ images of coexisting BCT and tubular particle strings. (F-H) 3D CLSM reconstructions of particle strings from 2D images in a $z$-stack $\left(4.4 \times 4.4 \times 6.2 \mu \mathrm{m}^{3}\right)($ see ESI Video S5†). All the structures formed are fully reversible, and the colloidal dispersions relax to their initial fluid-like disordered state after the field is turned off. 
site microgels at certain aspect ratios formed well-defined tubular structures as shown in our recent study. ${ }^{47}$ As the bowl-shaped particles are slightly anisotropic, these first observations indicate that field-directed tubular self-assembly may not be limited to ellipsoids but could as well occur when more complex anisotropic colloids are polarized in an AC field.

\section{Conclusion}

This study has demonstrated that we can successfully extended post-processing methods developed for polystyrene or poly(methylmethacrylate) lattices to responsive composite microgels. Stretching, faceting or opening up the originally spherical particles enables us to control the eccentricity, angularity and shape of the resulting particles. The fact that the outer microgel shell retains its thermoresponsive nature after post-processing results in a number of highly promising features. Temperature can be used to externally control and change the eccentricity, angularity and shape of the particles. Moreover, this also has consequences for the resulting interaction potential as temperature can be used to adjust the softness of the shell and induce an attraction above the volume phase transition temperature. This provides us with new opportunities to investigate the effects of shape and interaction anisotropy on the phase diagram of complex colloids. Combined with an external field, we have already seen that new self-assembly patterns emerge, and having a library of particles with tuneable size, shape and interaction potential will allow us to explore analogies between biological self-assembly processes and those present in complex synthetic colloids. Dynamic arrest in dense particle suspensions is another promising area where these particles provide new insights. In particular the bowl shaped particles where we can monitor not only the center of mass position and motion but also orientation and rotational motion offer exciting insights into the local structure and dynamics. While microgels have already been used to explore different arrest scenarios such as glass formation and jamming, ${ }^{75}$ bowl-shaped particles now further enlarge the scope for future studies to tuneable anisotropic particles that allow us to resolve orientational dynamics and thus directly determine the existence of a jamming transition.

\section{Acknowledgements}

We gratefully acknowledge financial support from the Swedish Research Council VR (project grant 621-2011-4338), the science faculty of Lund University and the European Research Council (ERC-339678-COMPASS) and the Knut and Alice Wallenberg Foundation (project grant KAW 2014.0052). The authors thank Dr Hervé Dietsch for the SEM measurements. Dr Vikash Malik and Liliane Ackermann-Hirshi are acknowledged for their respective help with the TEM measurements and the synthesis of some of the particles.

\section{References}

1 S. C. Glotzer and M. J. Solomon, Nat. Mater., 2007, 6, 557.

2 M. J. Solomon, Curr. Opin. Colloid Interface Sci., 2011, 16, 158.

3 H. Maeda and Y. Maeda, Phys. Rev. Lett., 2003, 90, 18303.

4 M. J. Solomon and P. T. Spicer, Soft Matter, 2010, 6, 1391.

5 A. Kuijk, A. van Blaaderen and A. Imhof, J. Am. Chem. Soc., 2011, 133, 2346.

6 A. Donev, I. Cisse, D. Sachs, E. Variano, F. H. Stillinger, R. Connelly, S. Torquato and P. M. Chaikin, Science, 2004, 303, 990.

7 A. Mohraz and M. J. Solomon, Langmuir, 2005, 21, 5298.

8 M. Radu, P. Pfleiderer and T. Schilling, J. Chem. Phys., 2009, 131, 164513.

9 S. Sacanna and D. J. Pine, Curr. Opin. Colloid Interface Sci., 2011, 16, 96.

10 D. Mukhija and M. J. Solomon, Soft Matter, 2011, 7, 540.

11 S. N. Fejer, D. Chakrabartia and D. J. Wales, Soft Matter, 2011, 7, 3553.

12 G. Odriozola, J. Chem. Phys., 2012, 136, 134505.

13 A. F. Demirors, P. M. Johnson, C. M. van Kats, A. van Blaaderen and A. Imhof, Langmuir, 2010, 26, 14466.

14 M. Marechal and M. Dijkstra, Soft Matter, 2011, 7, 1397.

15 J. D. Forster, J.-G. Park, M. Mittal, H. Noh, C. F. Schreck, C. S. O'Hern, H. Cao, E. M. Furst and E. R. Dufresne, ACS Nano, 2011, 5, 6695.

16 S. Andela, B. Behnaz and C. Angelo, J. Phys. Chem. B, 2011, 115, 7182.

17 M. Marechal, R. J. Kortschot, A. F. Demirors, A. Imhof and M. Dijkstra, Nano Lett., 2010, 10, 1907.

18 E. K. Riley and C. M. Liddell, Langmuir, 2010, 26, 1164811656.

19 S. Sacanna, W. T. M. Irvine, P. M. Chaikin and D. J. Pine, Nature, 2010, 464, 575-578.

20 S. Sacanna, W. T. M. Irvine, L. Rossi and D. J. Pine, Soft Matter, 2011, 7, 1631.

21 E. Y. K. Fung, K. Muangnapoh and C. M. L. Watson, J. Mater. Chem., 2012, 22, 10507.

22 L. Rossi, S. Sacanna, W. T. M. Irwine, P. M. Chaikin, D. J. Pine and A. P. Philipse, Soft Matter, 2011, 7, 4073.

23 S. Torquato and Y. Jiao, Nature, 2009, 460, 876.

24 A. Haji-Akbari, M. Engel, A. S. Keys, X. Zheng, R. G. Petschek, P. Palffy-Muhoray and S. C. Glotzer, Nature, 2009, 462, 773.

25 Hydrogel Micro and Nanoparticles, ed. L. A. Lyon and M. J. Serpe, Wiley VCH, Weinheim, Germany, 2012.

26 J. Z. Wu, G. Huang and Z. B. Hu, Macromolecules, 2003, 36, 440-448.

27 M. Rasmusson, A. Routh and B. Vincent, Langmuir, 2004, 20, 3536.

28 A. Zaccone, J. J. Crassous, B. Béri and M. Ballauff, Phys. Rev. Lett., 2011, 107, 168303.

29 A. Zaccone, J. J. Crassous and M. Ballauff, J. Chem. Phys., 2013, 138, 104908. 
30 J. McParlane, D. Dupin, J. M. Saunders, S. Lally, S. P. Armes and B. R. Saunders, Soft Matter, 2012, 8, 6239-6247.

31 J. J. Crassous, P. E. Millard, A. M. Mihut, F. Polzer, M. Ballauff and P. Schurtenberger, Soft Matter, 2012, 8, 1648.

32 D. Go, T. E. Kodger, J. Sprakel and A. J. C. Kuehne, Soft Matter, 2014, 10, 8060.

33 T. Ngai, S. H. Behrens and H. Auweter, Chem. Commun., 2005, 331-333.

34 B. Brugger, S. Rütten, K.-H. Phan, M. Möller and W. Richtering, Angew. Chem., Int. Ed., 2009, 48, 3978-3981.

35 S. Schmidt, T. Liu, S. Rütten, K.-H. Phan, M. Möller and W. Richtering, Langmuir, 2011, 27, 9801-9806.

36 W. Richtering, Langmuir, 2012, 28, 17218-17229.

37 K. Geisel, L. Isa and W. Richtering, Langmuir, 2012, 28, 15770-15776.

38 M. Destribats, V. Lapeyre, M. Wolfs, E. Sellier, F. Leal-Calderon, V. Ravaine and V. Schmitt, Soft Matter, 2011, 7, 7689-7698.

39 A. M. Mihut, A. P. Dabkowska, J. J. Crassous, P. Schurtenberger and T. Nylander, ACS Nano, 2013, 7, 10752-10763.

40 S. Schmidt, M. Zeiser, T. Hellweg, C. Duschl, A. Fery and H. Möhwald, Adv. Funct. Mater., 2010, 20, 3235-3243.

41 Z. K. Zhang, N. Krishna, M. P. Lettinga, J. Vermant and E. Grelet, Langmuir, 2009, 25, 2437.

42 M. Hoffmann, M. Siebenbuerger, L. Harnau, M. Hund, C. Hanske, Y. Lu, C. S. Wagner, M. Drechsler and M. Ballauff, Soft Matter, 2010, 6, 1125.

43 C. Dagallier, H. Dietsch, P. Schurtenberger and F. Scheffold, Soft Matter, 2010, 6, 2174.

44 V. Städele, U. Gasser and H. Dietsch, Soft Matter, 2012, 8, 4427-4431.

45 J. J. Crassous, A. M. Mihut, H. Dietsch, O. Pravaz, L. Ackermann-Hirschi, A. M. Hirt and P. Schurtenberger, Nanoscale, 2014, 6, 8726-8735.

46 J. J. Crassous, H. Dietsch, P. Pfleiderer, V. Malik, A. Diaz, L. A. Hirshi, M. Drechsler and P. Schurtenberger, Soft Matter, 2012, 8, 3538.

47 J. J. Crassous, A. M. Mihut, E. Wernersson, P. Pfleiderer, J. Vermant, P. Linse and P. Schurtenberger, Nat. Commun., 2014, 5, 5516, DOI: 10.1038/ncomms6516.

48 K. M. Keville, E. I. Franses and J. M. Caruthers, J. Colloid Interface Sci., 1991, 144, 103-126.

49 C. C. Ho, A. Keller, J. A. Odell and R. H. Ottewill, Colloid Polym. Sci., 1993, 271, 469.

50 T. Tanaka, Y. Komatsu, T. Fujibayashi, H. Minami and M. Okubo, Langmuir, 2010, 26, 3848.

51 S. H. Im, U. Y. Jeong and Y. N. Xia, Nat. Mater., 2005, 4, 671.

52 D. Feng, D. Marshburn, D. Jen, R. J. Weinberg, R. M. Taylor and A. Burette, J. Neurosci., 2007, 27, 12757-12760.
53 J. A. Champion, Y. K. Katare and S. Mitragotri, Proc. Natl. Acad. Sci. U. S. A., 2007, 104, 11901.

54 Y. Han, A. M. Alsayed, M. Nobili, J. Zhang, T. C. Lubensky and A. G. Yodh, Science, 2006, 314, 626-630.

55 D. Mukhija and M. J. Solomon, J. Colloid Interface Sci., 2007, 314, 98.

56 M. Basavaraj, J. Fransaer and J. Vermant, Langmuir, 2009, 25, 2718.

57 J. P. Singh, P. P. Lele, F. Nettesheim, N. J. Wagner and E. M. Furst, Phys. Rev. E: Stat. Phys., Plasmas, Fluids, Relat. Interdiscip. Top., 2009, 79, 050401.

58 M. Mittal and E. M. Furst, Adv. Funct. Mater., 2009, 19, 3271.

59 B. Madivala, S. Vandebril, J. Fransaer and J. Vermant, Soft Matter, 2009, 5, 1717.

60 S.-H. Kim, A. D. Hollingsworth, S. Sacanna, S.-J. Chang, G. Lee, D. J. Pine and G.-R. Yi, J. Am. Chem. Soc., 2012, 134, 16115-16118.

61 J. J. Crassous, C. N. Rochette, A. Wittemann, M. Schrinner and M. Ballauff, Langmuir, 2009, 25, 7862.

62 P. Pfleiderer and T. Schilling, Phys. Rev. E: Stat. Phys., Plasmas, Fluids, Relat. Interdiscip. Top., 2007, 75, 020402.

63 M. Letz, R. Schilling and A. Latz, Phys. Rev. E: Stat. Phys., Plasmas, Fluids, Relat. Interdiscip. Top., 2000, 62, 5173.

64 G.-C. Cho, J. Dodds and J. C. Santamarina, J. Geotech. Geoenviron. Eng., 2006, 132, 591-602.

65 Z. Zhang, N. Xu, D. T. N. Chen, P. Yunker, A. M. Alsayed, K. B. Aptowicz, P. Habdas, A. J. Liu, S. R. Nagel and A. G. Yodh, Nature, 2009, 459, 230-233.

66 D. Paloli, P. S. Mohanty, J. J. Crassous, E. Zaccarelli and P. Schurtenberger, Soft Matter, 2013, 9, 3000-3004.

67 S. Gangwal, O. J. Cayre and O. D. Velev, Langmuir, 2008, 24, 13312-13320.

68 S. O. Lumsdon, E. W. Kaler and O. D. Velev, Langmuir, 2004, 20, 2108-2116.

69 K. Chaudhary, J. J. Juárez, Q. Chen, S. Granick and J. A. Lewis, Soft Matter, 2014, 10, 1320-1324.

70 A. A. Shah, H. Kang, K. L. Kohlstedt, K. H. Ahn, S. C. Glotzer, C. W. Monroe and M. J. Solomon, Small, 2012, 8, 1551-1562.

71 C. Zhifeng, L. Fuhua, Z. Zexin and M. Yu-qiang, Soft Matter, 2013, 9, 11392-11397.

72 D. Nagao, M. Sugimoto, A. Okada, H. Ishii, M. Konno, A. Imhof and A. van Blaaderen, Langmuir, 2012, 28, 65466550 .

73 P. S. Mohanty, A. Yethiraj and P. Schurtenberger, Soft Matter, 2012, 8, 10819-10822.

74 S. Nojd, P. S. Mohanty, P. Bagheri, A. Yethiraj and P. Schurtenberger, Soft Matter, 2013, 9, 9199-9207.

75 A. Ikeda, L. Berthier and P. Sollich, Soft Matter, 2013, 9, 7669. 